\title{
Focus on Staff
}

\section{Oncology Education for Nurse Practitioners and Physician Assistants}

When nurse practitioner (NP) Gillian Serino, APRN-BC, began working at Commonwealth Hematology Oncology, the largest private oncology practice in New England, she went in with virtually no experience in hematology/oncology. "Initially, I followed two physicians for approximately 6 months. I did not see any patients independently at that time," Serino says. "I also worked with one of the nurse practitioners reviewing classes of medications weekly, as well." A combination of on-the job training and self-education using a combination of medical and nursing materials, including resources provided by the Oncology Nursing Society (ONS), has helped Serino to progress quickly, and she plans to take the Advanced Oncology NP Exam soon. But, she says, "We do not have a specific curriculum that we follow for training new oncology NPs in our practice."

Many NPs and physician assistants (PAs) complete comprehensive general nursing and medical programs, but receive little oncology-specific training to allow them to specialize in this unique area. In many programs, one does not need to be a registered nurse before becoming an NP, so the educational background can vary widely. "I felt strongly about having a foundation in primary care before entering a specialty practice, and I feel that the time I spent in primary care afforded me a foundation from which to more easily transition into a specialty," says Serino "A lot of my practice is spent managing the affects of medications which have a systemic effect on the whole patient, so knowing normal versus abnormal findings (which I felt more confident about after having spent caring for patients who were generally well in a primary care setting) built my confidence in an oncology setting and ultimately makes me a more competent health care provider." But not all NPs and PAs have that experience before deciding to pursue an oncology specialty, so a comprehensive training program is to their benefit.

Like Serino, University of Michigan Cancer Center Lead NP and member of the ONS Board of Directors Peg Esper has noted curriculum gaps in how her center orients new NPs and PAs. "There are many critical pieces of information that one needs to possess to work in the oncology specialty arena," Esper says. "These include cancer biology, genetics, knowledge of chemotherapy and biologic therapy mechanisms of action and side effects, oncology emergencies, symptom management and palliative care, and information on specific types of cancer."

In addition, Esper says, oncology NPs and PAs need to be knowledgeable in the areas of clinical trials and research methods. "Fortunately, all NPs are required to have Master's degrees and so they do get basic research as part of their curriculum, but they still need to understand how cancer clinical trials are run," she says. "There are also the procedures that are unique to oncology that most graduate programs would not have included: bone marrow biopsies, lumbar punctures, and so on. The fact that many NPs have worked for a while as nurses before becoming NPs increases the chance that they will at least be familiar with some of these procedures, but being able to do them safely and proficiently is still something that must be documented and part of an oncology orientation."

David Coniglio, MPA, PA-C, is an assistant professor in the Department of Community and Family Medicine at Duke University Medical Center who plays a central role in the PA program. "Most PA programs have a 2-year curriculum," he explains. "The didactic year curriculum includes coursework in the basic medical sciences, clinical medicine, surgery, pharmacology, radiology and laboratory medicine, behavioral medicine, principles of evidence-based medicine, and others. The clinical year will typically include a number of required clinical rotations such as surgery, internal medicine, behavioral medicine, pediatrics, obstetrics and gynecology, emergency medicine, and others; and also includes electives such as hematology/oncology.”

To meet the unique needs of NPs and PAs specializing in oncology, the American Society of Clinical Oncology's (ASCO) Clinical Practice Committee (CPC) and ONS have formed a taskforce with the dual goals of (1) attracting PAs and NPs to a career in oncology at the beginning of their professional development and (2) equipping them with the specialized knowledge they need to be successfully integrated into the collaborative practice model. A partnership with the American Academy of Physician Assistants is also planned.

Says ASCO CPC Past Chair Peter Yu, MD, "If we can introduce nonphysician practitioners such as NPs and PAs to oncology as a part of their practicum experience, we will open new doors for them to explore. A flexible curriculum that provides continuing education while accommodating the time constraints of the midlevels is needed."

To that end, "We are developing what we believe will be an intensive learning course that can be used to help provide both the didactic and clinical experiences that will provide just this type of orientation," Esper says. "The Oncology 


\section{Additional NP and PA Education Resources}

\section{Web sites}

- American Academy of Physician Assistants: www. aapa.org

- National Commission on Certification of Physician Assistants: www.nccpa.net

- Oncology Nursing Society:www.ons.org

- Physician Assistant Accreditation Review Commission: www.arc-pa.org

- Physician Assistant Education Association: www.paeaonline.org

- American Academy of Nurse Practitioners: www.aanp.org

\section{Books}

- Oncology Medical Knowledge Self-Assessment Program. Alexandria, VA, ASCO

- Lin EM: Advanced Practice in Oncology Nursing: Case Studies and Review. Pittsburgh, PA, Oncology Nursing Society, 2001

- Camp-Sorrell D, Hawkins RA: Clinical Manual for the Oncology Advanced Practice Nurse. Pittsburgh, PA, Oncology Nursing Society, 2006

Nursing Society has a vast amount of resource material available on their Web site. They also host an Advance Practice Nursing conference in the fall of each year. While this is a great help to NPs currently working or interested in working in oncology, we are trying to develop something that will be available in more of an 'on-demand' fashion."

Yu describes a vision for Web-based education modules. "One concept under consideration is a series of educational modules to be developed, each focusing on a cancer category such as breast cancer, hematological diseases or clinical research," says Yu. "Current CPC Chair Therese Mulvey and I have integrated NPs into our practices and we are most familiar with NPs as nonphysician practitioners. This has led to an early focus on NPs, although PAs are an equally important resource for oncologists.

Serino supports the formulation of a standardized curriculum to educate those entering the oncology specialty. "Looking forward, I hope that NPs are leaders in the development of a curriculum that would affect their training and ultimately their role in oncology," she says. "I believe we serve a vital role in our patients' lives. We contribute to a broader range of knowledge for treating and caring for patients and, when utilized appropriately by both physicians and nurses, we serve as an integral part of the patient's health care team."

Elective hematology/oncology education options exist in many PA training programs. "The PA program at Duke has an instructional unit in the clinical medicine course which offers instruction in hematologic and oncologic disease processes; additional oncology specific lecture content is offered in the surgery, diagnostic methods and pharmacology courses," Coniglio says. "Students may elect a 4-week clinical rotation in the second year. In this clinical elective, students are exposed to a variety of academic and community models of oncology care." He also points out that there exists one postgraduate training program for PAs interested in oncology.

However, Coniglio recognizes that there are numerous opportunities to expand the role of nonphysician providers in oncology care. "Increasing attention should be paid to developing additional oncology-specific continuing medical education material for nonphysician providers, and I think this is a place where ASCO is in an excellent position to contribute expertise," he says. "PAs practice collaboratively with physicians, and currently work in all aspects of oncology care: medical, surgical, radiation, and transplant, and in pediatric and adult settings. Educational models should reflect the unique nature of this collaborative practice, and should be inclusive of the variety of practice settings for inpatient and outpatient care, in community practices as well as academic medical centers."

The ultimate goal of developing a standardized curriculum for oncology NPs and PAs is a flexible solution that can be customized to individual practitioners and practices. "Practice settings vary from small to large, but an urgent need for more health care providers to handle the volume of patients is strikingly evident," says Yu. "Oncologists practice in different ways and how each practice works with their midlevel providers will depend on the needs of that practice. Some practices do not see hematology patients, or a nonphysician clinician may find that he or she does not feel comfortable with their background knowledge of treating prostate cancer for example. The modules would allow a means to remedy that."

DOI: 10.1200/JOP.0755501 